An approach to similarity measurement of absence-presence data: the case that common zeros matter

Non Peer-reviewed author version

EGGHE, Leo \& ROUSSEAU, Ronald (2004) An approach to similarity measurement of absence-presence data: the case that common zeros matter. In: Journal of Information Science, 30(6). p. 509-519.

DOI: $10.1177 / 0165551504047827$

Handle: http://hdl.handle.net/1942/757 
An approach to similarity measurement of absence-presence data:

The case that common zeros matter

\section{Leo Egghe}

LUC, Universitaire Campus, B-3590 Diepenbeek, Belgium

\& UA, IBW, Universiteitsplein 1, B-2610 Wirijk, Belgium

Email: leo.egghe@luc.ac.be.

\section{Ronald Rousseau}

KHBO, IWT, Zeedijk 101, B.8400 Oostende, Belgium

\& UA, IBW, Universiteitsplein 1, B-2610 Wilrijk, Belgium

Email: ronald.rousseau@khbo.be 


\begin{abstract}
Similarity between objects (documents, persons, answers to a questionnaire, etc.) is generally determined through relations between representations of these objects. In the case of binary representations the presence of a property (e.g., an index term) carries a weight of one, the absence a weight of zero. In many similarity studies common zeros are ignored. This situation is called the zero insensitive case. In this article, however, we study the zero sensitive case. Clearly, answers to binary questionnaires (yes-no, encoded as 1-0) are zero sensitive, as people who answer 'no' to the same questions are more similar. We present a wish list for such a zero sensitive approach to similarity. Making a difference between common zeros and common ones leads to an 'identitysimilarity' theory. Hence, we move beyond a pure similarity theory. Three approaches to the problem of similarity measurement of presence-absence data, where common zeros matter are presented. In each case a coding approach is used, leading to new representations, which then lead to a similarity ranking. Examples of functions respecting these rankings are given.
\end{abstract}

Keywords: Zero-sensitive similarity, absence-presence data, differences between identical representations, 


\section{Introduction}

In a previous article [1] we studied similarity measurement for absence-presence data. Similarity between documents is determined by comparing document representations. In the case of binary representations the presence of an index term (keywords or phrases) carries a weight of one, the absence a weight of zero. In information retrieval and in overlap studies it is customary not to consider common zeros when determining the similarly between documents, or more precisely, document representations [2]. Indeed, keywords or phrases that do not occur in the two documents under consideration have no influence on the similarity between these two documents. Economic articles are not more similar if the term "Big Bang" is absent in both. This situation is called the zero insensitive case. That was the case studied in our previous article. In this article we will study the zero sensitive case. Clearly, answers to binary questionnaires (yes-no, encoded as 1-0) are zero sensitive, as people who answer 'no' to the same questions are more similar. Probably, two authors working in the same field, who are never co-cited, or never collaborated with a third colleague are more similar than in the case one had been co-cited and the other not. Further, when doing a search in a binary-indexed database using the NOT-operator declares two documents to be more similar if they do not contain the NOT-ed term. As in [1] we emphasize the fact that it is irrelevant in which order document representations $r$ and $s$ for similarity studies are considered by referring to $D$ $\{r, s\}$ as a duo, a word that has no "rank" connotations. 


\section{A wish list}

In the zero sensitive case we would like to construct a similarity theory with the following properties:

P1 Adding two common 1s makes two non-identical arrays (strictly) more similar:

$$
\{(x, \ldots, x),(y, \ldots, y)\} \rightarrow\{(x, \ldots, x, 1),(y, \ldots, y, 1)\} \text { increases similarity }
$$

P2 Adding two common 0s makes two non-identical arrays (strictly) more similar:

$$
\{(x, \ldots, x),(y, \ldots, y)\} \rightarrow\{(x, \ldots, x, 0),(y, \ldots, y, 0)\} \text { increases similarity }
$$

P3 Adding a (0-1) to a duo makes it (strictly) less similar:

$$
\{(\mathrm{x}, \ldots, \mathrm{x}),(\mathrm{y}, \ldots, \mathrm{y})\} \rightarrow\{(\mathrm{x}, \ldots, \mathrm{x}, 0),(\mathrm{y}, \ldots, \mathrm{y}, 1)\} \text { decreases similarity }
$$

P4 Replacing a (0-1) by a (0-0) makes the arrays (strictly) more similar:

$$
\{(x, \ldots, 0, x),(y, \ldots, 1, y)\} \rightarrow\{(x, \ldots, 0, x),(y, \ldots, 0, y)\} \text { increases similarity }
$$

P5 Replacing a (0-0) by a (1-1) makes the arrays (strictly) more similar:

$$
\{(x, \ldots, 0, x),(y, \ldots, 0, y)\} \rightarrow\{(x, \ldots, 1, x),(y, \ldots, 1, y)\} \text { increases similarity }
$$

or the weaker version: 
P5a: Replacing a $(0-0)$ by a (1-1) does not alter the similarity between the two arrays:

$\{(x, \ldots, 0, x),(y, \ldots, 0, y)\} \rightarrow\{(x, \ldots, 1, x),(y, \ldots, 1, y)\}$ does not alter similarity

Preferably, we would like to represent this similarity theory using a Lorenz curve approach. Note that the difference between P5 and P5a is that in P5a common Os and common 1s are considered to have the same impact on the similarity of the duo under consideration: the occurrence of a common 0 or a common 1 makes the items in a duc in the same way more similar. According to P5, however, common 1s make a duo more similar than common 0s (introducing a kind of property weighting). We think that both considerations are meaningful, depending on the application one has in mind. Note that P5 implies that identical arrays with at least one set of corresponding 0 s are not considered perfectly similar anymore, because otherwise repiacing a $(0-0)$ by $(1-1)$ would not lead to a strict increase in similarity. This means that introducing weights brings the theory beyond a pure similarity theory. It becomes an identity-similarity' theory. Note also that requirements P4 and P5 (and hence certainly P5a) imply that replacing a (0-1) by (1-1) should make two arrays more similar.

We consider our wish list as a set of logical requirements. We admit though that other requirements are possible [3]. One could also imagine a similarity theory where not all of these requirements are satisfied (it is just a wish list). The main point is that when discussing similarity in general terms authors should clearly 
state which requirements they imply. It is only then that the problem of the best measure for a given study can be brought up for discussion in a meaningful way.

We present three approaches to the problem of simitarity measurement of presence-absence data, where common zeros matter. In each case the duo will be encoded, i.e., a new representation is used, and then these new representations lead to a similarity ranking. Examples of functions respecting these rankings will be given.

The following standard contingency table is used.

\begin{tabular}{|l|l|l|}
\hline Array $r$ & presence & absence \\
\hline Array s & & \\
\hline presence & $\begin{array}{l}p: \text { number of matches in which a } \\
\text { given property is present }\end{array}$ & $\begin{array}{l}\text { k: number of cases for which a } \\
\text { property is present in array } r \text {, and }\end{array}$ \\
\hline absence & $\begin{array}{l}\text { l: number of cases for which a } \\
\text { propenty is present in array r, and } \\
\text { absent in array s }\end{array}$ & $\begin{array}{l}\text { m: number of matches in which a } \\
\text { given property is absent }\end{array}$ \\
\hline
\end{tabular}

$N=p+m+k+1$

\section{A first approach: the simple binary model}

In the simple binary model we only take into account if zeros or ones correspond or not. Concretely: if zeros or ones correspond this is encoded as a 1, if they do not it is encoded as a 0 . For example, if the duo $D=\{r, S\}$ consists of $r=$ $(1,1,1,1,0,0)$ and $s=(0,0,1,1,0,1)$ Then $D$ is encoded as $\left[\begin{array}{lllll}0 & 0 & 1 & 1 & 1\end{array}\right]$. As the order in which properties are censidered should not matter, this coding is equivalent to $\left[\begin{array}{lllll}1 & 1 & 1 & 0 & 0\end{array}\right]$. In other words: the length of the array, $N$, (here $\left.N=6\right)$ 
and the number of common symbols, $d=p+m$, (here $d=3$ ) contain ali information. In this situation there is one obvious similarity measure, namely $\mathrm{d} / \mathrm{N}$, although applying any monotone increasing function would (at least in theory) also be acceptable. Note that $\mathrm{d} / \mathrm{N}$ is the fraction of ones in the encoded form, i.e., the fraction of common symbols (zeros or ones). The fraction $\mathrm{d} / \mathrm{N}$ is also known as the simple matching coefficient. It is not difficult to introduce a Lorenz curve and a Lorenz similarity partial order corresponding to the simple binary model. It suffices to use the classical Lorenz curve for the encoded array. This is illustrated in Fig.1 for $D=\{r=(1,1,1,1,0,0), s=(0,0,1,1,0,1)\}$, encoded as $\left[\begin{array}{lllll}1 & 1 & 1 & 0 & 0\end{array}\right]$.

Insert Fig. 1 about here

Definition: SB-equivalent duos

Two duos are said to be SB-equivalent, i.e. equivalent for the simple binary model, if and only if the Lorenz curves derived from their SB-encoded form coincide.

From now on, equivalent duos will be considered to be the same. Hence, a symbol such as $D=\{r, s\}$ will represent the equivalence class of $\{r, s\}$. In the set of equivalent duos we say that $D 1<1, D 2$, meaning that $D 1$ is a less similar duo than $\mathrm{D} 2$, if the Lorenz curve of D1 is situated above the Lorenz curve of D2. The relation D1 S, D2 then means that D1 and D2 may also be equivalent. The order relation $s_{1}$ is a total order for equivalence classes, because Lorenz curves of the 
type studied here, never cross. Indeed, the Lorenz curve is completely determined by the point with coerdinates $(d / N, 1)$.

How does the simple binary approach fare with respect to the wish list? Adding two common ones or two common zeros makes arrays more similar: $\mathrm{d} / \mathrm{N}$ becomes $(d+1) /(N+1)$, which is strictly larger. So the simple binary model satisfies P1 and P2. Adding a $(0-1)$ decreases the similarity as $d / N>d /(N+1)$. Replacing a $(0,1)$ by a $(0,0)$ makes arrays mere-similar, as $d / N$ becomes $(d+1) / N$. Hence also P4 is satisfied. Further, only the weaker version P5a is satisfied, as zeros and ones play equivalent roles. Finally, we already introduced a Lorenz curve associated with the simple binary model.

Recall that a classical Lorenz curve is replication invariant, i.e. replicated duos are equivalent [4]. The case where no two symbols coincide, encoded as an allzero array, does not lead to a regular Lorenz curve, yet it can be represented by the line segment connecting the origin with the left upper corner, followed by the line segment connecting the left upper corner with the right upper corner.

The Gini similarity measure corresponding to this Lorenz curve is nothing but twice the area above the curve. This is equal to $\mathrm{d} / \mathrm{N}$, leading to a convenient interpretation of this measure. Interpreted othenvise, this measure is nothing but the normalized complement of the Hamming distance (the number of symbols that disagree) between two arrays [5]. 
The reciprocal of the coefficient of variation, another acceptable measure, is $\sqrt{\frac{d}{N-d}}=\frac{1}{\sqrt{1-\frac{d}{N}}}$ (the second formula clearly shows that this is just a monotone transformation of $d / N$ ), while the similarity-normalized length of the Lorenz curve is $\frac{2-L}{2-\sqrt{2}}$ (here $L$ denotes the length of the Lorenz curve). One should wonder, however, why using measures like this, when there exists a measure that is both simple and exact in its description of similarity according to the simple binary model. Generally, any monotone increasing function of $d / N$ is again an acceptable similarity measure. Examples of such increasing functions are the Tindices:

$$
\frac{d}{d+\beta(N-d)}
$$

with $\beta>0$. This family of functions includes the Sokal \& Sneath coefficient $(\beta=$ $1 / 2)$, and the Rogers \& Tanimoto coefficient $(\beta=2)[6]$. A proof is given in the appendix.

\section{A second approach: reduction to the zero-insensitive case}

In this approach we again declare common zeros to be completely equivalent to common ones (as we did in the previous one). Common zeros are first rewritten as common ones, and then the approach taken for the zero-insensitive case is applied. For example $D=\{r=(1,1,1,1,0,0), s=(0,0,1,1,0,1)\}$, is first rewritten as: $D^{*}=\left\{r^{*}=(1,1,1,1,1,0), s^{*}=(0,0,1,1,1,1)\right\}$, and then represented by the similarity 
Lorenz curve joining the points $(0,0)-(2 / 6,8 / 20)-(5 / 6,5 / 20)-(1,0)$ (see Fig.2). This approach will be called (in short) the reduction approach.

Insert Fig. 2 about here

Definition: Zl-equivalent duos

Two duos are Zl-equivalent if their Lorenz zero-insensitive curves coincide.

We know, from [1] that the reduction approach leads to a partial order, which we will denote by $\leq_{2}$. We further already know [1] that also these Lorenz curves are replication invariant.

If D1 $<_{2} D 2$ then D1 $<_{1} D 2$. Indeed D1 $<_{2} D 2$ implies that the Jaccard index of D1, denoted as $J(D 1)$, is strictly smaller than $J\left(D_{2}\right)$. Hence $d_{1} / N_{1}<d_{2} / N_{2}$, which is equivalent to $\mathrm{D} 1<\mathrm{D} 2$. For the same reason equivalent duos according to the reduction approach correspond to equivalent duos according to the simple binary model. The opposite, though, is not true. Consider, for instance, D1 $=\left\{\mathrm{r}_{1}=\right.$ $\left.(1,1,1,0,0,0), s_{1}=(1,0,0,1,1,1)\right\}$ and $D 2=\left\{r_{2}=(1,1,1,1,1,0), s_{2}=(1,0,0,0,0,1)\right\}$. According to the simple binary model D1 and D2 are both represented as $\left[\begin{array}{lllllllll}1 & 0 & 0 & 0\end{array}\right.$ 0 0], hence are SB-equivalent. Yet, D1 and D2 are incomparable in the reduction model, as illustrated in Fig.3.

Insert Fig.3 about here 
Examples of acceptable similarity measures

As this approach reduces the problem to the zero-insensitive case, we may use those measures known to be applicable in this case [1]. Examples of acceptable measures are the classical similarity measures such as the Jaccard index (equal to the Gini similarity index), Dice's coefficient, Salton's cosine measure, and the adapted Simpson index. Expressed in the notation of this article they have the following mathematical expressions.

The Jaccard index of a duo $D$ is defined here as:

$$
J(D)=\frac{d}{N}
$$

Dice's coefficient of the duc $D=\{r, s\}$ is:

$$
\operatorname{Dice}(D)=\frac{2 d}{\rho+\sigma}
$$

where $\rho=p+m+l$ is the number of $1 \mathrm{~s}$ in $r^{*}$ and $\sigma=p+m+k$ is the number of $1 \mathrm{~s}$ in $\mathrm{s}^{*}$.

Salton's cosine measure of $D$ becomes:

$$
\cos (D)=\frac{d}{\sqrt{\rho \sigma}}
$$

where $\rho$ and $\sigma$ have the same meaning as for the Dice coefficient. 
Finally the adapted Simpson index of D is:

$$
S(D)=\frac{\rho \cdot \sigma}{N(\rho+\sigma-2 d)}
$$

Any strictly increasing function of these measures is again an acceptable measure in the reduction approach.

The attentive reader may have noticed that we have not yet discussed if the reduction approach satisfies the requirements $\mathrm{P} 1-\mathrm{P} 5$ (a). The reason is that it does not, at least it does not meet all the requirements. Let us discuss them one by one. First, because common 0 s are encoded as common 1s, we note that requirements $\mathrm{P}_{1}$ and $\mathrm{P}_{2}$ coincide. These requirements are satisfied by the reduction approach, as shown in our previous article [1]. Clearly, P5 cannot be met, and P5a is trivially satisfied. This leaves P3, about adding a (0-1) and P4 about changing a $(0-1)$ by common 0 s (here common 1s). P3 is not met as shown by the following example: we transform $D 1=\left\{r_{1}=(1,1,1,0,0,0), s_{1}=\right.$ $(1,0,0,1,1,1)\}$ to $D 3=\left\{r_{3}=(1,1,1,0,0,0,0), s_{3}=(1,0,0,1,1,1,1)\right\}$. Then the Lorenz curves of D1 and D3 cross, showing that D1 and D3 are not intrinsically comparable (see Fig. 4).

Insert Fig.4 here

Finally, P4 is satisfied in this approach. Replacing a (0-1) by common 1 s always leads to an intrinsically more similar situation. A proof is provided in the appendix. 


\section{Third approach: using radix 4 encoding}

In this approach common is are encoded as 3, common 0s as 2, different symbols as Os followed by one 1. A duo is then encoded as the number 'zero point' followed by the code numbers arranged in decreasing order, such as 0.3.32..20..01. All codes of the form 0.3...3 (any number of $3 \mathrm{~s}$ ) are declared equivalent (corresponding to perfect similarity). Similarly, all codes beginning with 0.0 are declared equivalent (corresponding to the case that not a single symbol corresponds). For example: $\mathrm{D}=\{r=(1,1,1,1,0,0), \mathrm{s}=(0,0,1,1,0,1)\}$ is encoded as: 0.3320001 , because there are two $1 \mathrm{~s}$ in common, one 0 in common and three symbols which are different in the two arrays.

As in the other approaches we declare two duos to be equivalent if they lead to the same code. This is true for all duos consisting of identical items, but also for, e.g., $D=\{r=(1,1,1,1,0,0), s=(0,0,1,1,0,1)\}$, and $D^{\prime}=\left\{r^{\prime}=(1,1,1,1,0,1)\right.$, $s^{\prime}=$ $(0,0,1,1,0,0)\}$. This approach again leads to a complete order on equivalence classes, denoted as $\leq_{3}$.

For similarity considered in this way, the requirements $\mathrm{P} 1, \mathrm{P} 2, \mathrm{P} 3, \mathrm{P} 4$ and $\mathrm{P} 5$ are all satisfied: adding common Os (and certainly 1s) yieids a larger code number, adding $0-1$ decreases the similarity, replacing $0-1$ by common 0 s yields a larger code number, replacing common 0s by common 1s also yields a larger code number. A drawback of this approach is that two identical arrays with at least one 
0 are not considered to be completely similar anymore. If they were then requirement P5, stating that replacing a $(0-0)$ by a (1-1) makes the arrays more similar, would not satisfied anymore.

Because only the symbols 3,2 and 1 and 0 are used these codes can be considered as numbers in the base or radix 4 number system. In this system, similar to the better known, binary number system, the number 0.31 corresponds to the decimal number $\frac{3}{4^{1}}+\frac{1}{4^{2}}=0.8125$, similarly 0.2201 corresponds to $\frac{2}{4^{1}}+\frac{2}{4^{2}}+\frac{0}{4^{3}}+\frac{1}{4^{4}}=0.62890625$. Note however that if two duos have at least one symbol in common, their decimal representation is at least 0.5 . Hence as a similarity measure we propose the decimal representation minus 0.5 , and the result multiplied by 2 . In this way 1 stays 1 , namely $(1-0.5) .2=1$, while 0.5 becomes $(0.5-0.5) .2=0$. This proposal has one small inconvenience, namely that the theory cannot be applied to arrays of length one, because then the duo $\{(0),(0)\}$ would have measure 0 . But who wants to study the similarity of arrays of length one?

This encodiny, and hence this approach to similarity, is not duplication invariant, and duplication increases similarity. In this sense one may say that the radix 4 approach is an approach based on absolute numbers, not on relative proportions as in the replication invariant cases. Indeed, D5 $=\{(0,0),(0,0)\}$ is encoded as $0.22 ;$ D5 $=\{(0,0),(1,0)\}$ is encoded as 0.201. Hence D6 $\leq_{3}$ D5. However, 
replicating D6 twice leads to $D 7=\{(0,0,0,0),(1,1,0,0)\}$, which is encoded as 0.22001 , so that $\mathrm{D} 6 \leq_{3} \mathrm{D} 5 \mathrm{~s}_{3} \mathrm{D} 7$.

We were not able to find a non-trivial Lorenz curve representation corresponding to the radix 4 approach. This is not surprising as traditional Lorenz curves are duplication invariant. Yet, connecting the origin to the point with as abscissa the decimal representation of the duo's code, and as ordinate the value one, yields in a trivial way - a kind of Lorenz curve corresponding with this encoding. As for the first approach the encoding - in decimal form - corresponds to the Gini similarity index of this Lorenz curve, but it is possible to consider other acceptable similarity measures, using the similarity equivalents of the coefficient of variation, the length of the Lorenz curve, the entropy measure and so on. As for the first approach, we do not consider these other measures of real practical value.

\section{Different shades of identity}

In the previous approach we crossed the thin line between a pure similarity theory and what we would like to call an 'identity-similarity' theory, as we made a distinction between different identical duos. From that point of view it certainly seems artificial to declare all arrays coded as "zero point any number of $3 \mathrm{~s}$ " to be 'identical'. The same is true for the $0.0 \ldots .01$ case. Of course, the first type of code represents identical arrays, so they are as similar as possible. The second type represents completely dissimilar arrays. Yet, taken these codes as they are, 
without the extra correction, makes it pessible to say that identical arrays with more 1s are more similar than identical arrays with less $1 \mathrm{~s}$. A similar remark goes for the dissimilar arrays. We will not go further here into the issue of "different shades of identity', as this is not a pure similarily theory anymore.

Notes

a) A generalization

If the data are not $0-1$ data but categorical data, with no relation at all between the categories, then the similarity of such a duo can be reduced to the first case, where a 1 represents the case that categories coincide, and a zero when they do

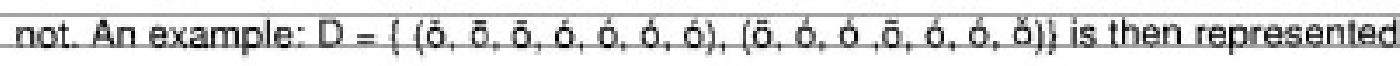
as $[0000110]=[1100000]$.

\section{b) Dichotomizing}

Any set of numerical data can be dichotomized in a low and high calegory. Then the absence-presence-similarity theory presented here can be applied.

c) Another look at the binary case.

In the simple binary model that we presented corresponding zeros as well as ones were encoded as is. One could imagine an encoding in which only common ones are encoded as $1 \mathrm{~s}$. Cemmon zeros are then treated as dissimilar symbols. The duo $D=(r, s\}$ consisting of $r=(1,1,1,1,0,0)$ and $s=(0,0,1,1,0,1)$ is then encoded as $\left[\begin{array}{llllll}0 & 0 & 1 & 1 & 0 & 0\end{array}\right]=\left[\begin{array}{llllll}1 & 1 & 0 & 0 & 0 & 0\end{array}\right]$. As before, $\mathrm{d} / \mathrm{N}$ (in the encoded 
form) seems a reasonable candidate similarity measure. Yet, this approach violates requirement $P 2$. Adding two common zeros reduces the similarity from $d / N$ to $d /(N+1)$. For this reason we reject this approach.

d) The zero-insensitive case

Requirements $\mathrm{P} 3$ and $\mathrm{P} 4$ were not studied in our previous article covering the zero-insensitive case. The counterexample and proof given here show that also in the zero-insensitive case P3 is not satisfied, while P4 always is.

e) Lorenz curves

We were able to extend the Lorenz curve approach (as in the reduction to the insensitive case), but then at least one of the requirements P1-P5 was not always satisfied. Details can be obtained from the authors. Anyhow, the Lorenz curves of the second approach must be altered as it is easy to see that adding common Os to such a Lorenz curve lifts the curve. This is an unwanted property as we want to lower this curve in a similarity theory. This shows that the Lorenz curve approach (at least without alterations) cannot be used for a similarity theory where common zeros are possible.

f) The idea of the radix 4 approach may also be applied to the case that common Os and common 1s are considered to be perfectly equal. It suffices to give the same encoding, e.g. 2, to both (making it a radix 3 approach). 


\section{Conclusion}

In this article we studied similarity for the zero sensitive case of absencepresence data. A wish list for such a zero-sensitive approach to similarity was drawn:

P1 Adding two common 1s makes two non-identical arrays (strictly) more similar.

P2 Adding two common 0s makes two non-identical arrays (strictly) more similar.

P3 Adding a (0-1) to a duo makes it (strictly) less similar.

P4 Replacing a ( $0-1)$ by a (0-0) makes the arrays (strictly) more similar.

P5 Replacing a $(0-0)$ by a (1-1) makes the arrays (strictly) more similar, or the weaker version:

P5a: Replacing a $(0-0)$ by a $(1-1)$ does not atter the similarity between the two arrays.

Two approaches were given where common 1 s and common 0s are treated in the same way, having the same impact on the similarity of the duo under consideration. The simple binary case leads to a similarity theory respecting requirements $\mathrm{P} 1$ to $\mathrm{P} 4$ of the wish list and P5a. Introducing different weights for common $1 \mathrm{~s}$ and common $0 \mathrm{~s}$, teads to an identity-similarity theory. Such a theory respects all requirements (P1 to P5) of the wish list. Examples of functions respecting the corresponding similarity rankings are given. 
Ultimately, any similarity theory is only useful when it helps to understand real-life examples. We invite our colleagues, not only in the information sciences, but also in the fields of ecology, sociology and computer sciences, to try out the approach presented in this article.

\section{References}

[1] L. Egghe and R. Rousseau, Classical retrieval and overlap measures satisfy the requirements for rankings based on a Lorenz curve, Information Processing and Management (2004, to appear).

[2] G. Salton and M.J. McGill, M.J., Introduction to modern information retrieval. (McGraw-Hill, New York, 1983).

[3] R.E. Tulloss, R.E., Assessment of similarity indices for undesirable properties and a new tripartite similarity index based on cost functions. In: M.E. Palm and I.H. Chapela, eds), Mycology in sustainable development: expanding concepts, vanishing borders (Parkway Publishers, Boone, NC, 1997) pp. 122-143.

[4] D. Nijssen, R. Rousseau and P. Van Hecke, The Lorenz curve: a graphical representation of evenness, Coenoses, 13(1) (1998) 33-38. 
[5] R.W. Hamming, Error detecting and error correcting codes, Bell Systems Technical Journal, 29, (1950) 147-160.

[6] P.H.A. Sneath and R.R. Sokal, Numerical taxonomy. (Freeman \& Co, San Francisco, 1973).

\section{Appendix}

Proposition 1

The function $\mathrm{T}=\frac{d}{d+\beta(N-d)}, \beta>0$, is a monotone increasing function of $\mathrm{d} / \mathrm{N}$.

Proof. The T-index can be rewritten as: $\frac{d / N}{d / N+\beta(1-d / N)}$. Writing $\times$ for $\mathrm{d} / \mathrm{N}$ gives: $T=\frac{x}{x+\beta(1-x)}$. Taking the derivative of $\mathrm{T}$ with respect to $\mathrm{x}$ gives: $T^{\prime}=\frac{\beta}{(x+\beta(1-x))^{2}}$. This expression is always positive, proving that $\mathrm{T}$ is a monotone increasing function of $\mathrm{d} / \mathrm{N}$.

Proposition 2

Replacing a (0-1) by common 1 s leads to a Lorenz curve representing an intrinsically more similar situation. 
Proof.

The similanity Lorenz curve is determined by the points with coordinates $S=\left(\frac{\rho-d}{N}, \frac{\rho-d}{\rho}\right)$ (this point is called the sub-top) and $T=\left(\frac{\rho}{N}, \frac{\sigma-d}{\sigma}\right)$ (the top). Here $d=p+m, p=p+m+1, \sigma=p+m+k$, and $N=p+m+k+1$. Replacing $(0-1)$ by common is leads either to a situation where $d^{\prime \prime}=p+m+1, p^{\prime}=p+m+1, \sigma^{\prime}$ $=p+m+k+1$, and $N^{\prime}=p+m+k+1$; or $d^{\prime}=p+m+1, \rho^{\prime}=p+m+1+1, \sigma^{\prime}=p+m+k$, and $N^{\prime}$ $=p+m+k+1$. In the first case the new sub-top and top are: $s_{1}-\left(\frac{\rho-d-1}{N}, \frac{p-d-1}{\rho}\right), T_{1}=\left(\frac{p}{N}, \frac{\sigma-d}{\sigma+1}\right)$, and the second case they become: $S_{2}=\left(\frac{p-d}{N}, \frac{p-d}{\rho+1}\right), T_{2}=\left(\frac{p+1}{N} \cdot \frac{\sigma-d-1}{\sigma}\right)$, Clearly, $T_{1}$ is situated under $T$, while $S$ and $S_{1}$ are situated on the same line through the origin. Similarly, $S_{2}$ is situated under $S_{1}$ while $T$ and $T_{2}$ are on the same line through the endpoint $E$ with coordinates $(1,0)$. This shows that the new Lorenz curve depicts an intrinsically more similar situation than the original one. The proof is illustrated by the transformation of $\mathrm{D} 1=\left\langle r_{1}=(1,1,1,0,0,0), \mathrm{s}_{1}=\{1,0,0,1,1,1)\right\} 10 \quad \mathrm{D} 4=\left\{r_{4}=\right.$ $\left.(1,1,1,0,0,1), \mathrm{s}_{d}=(1,0,0,1,1,1)\right\}$. 


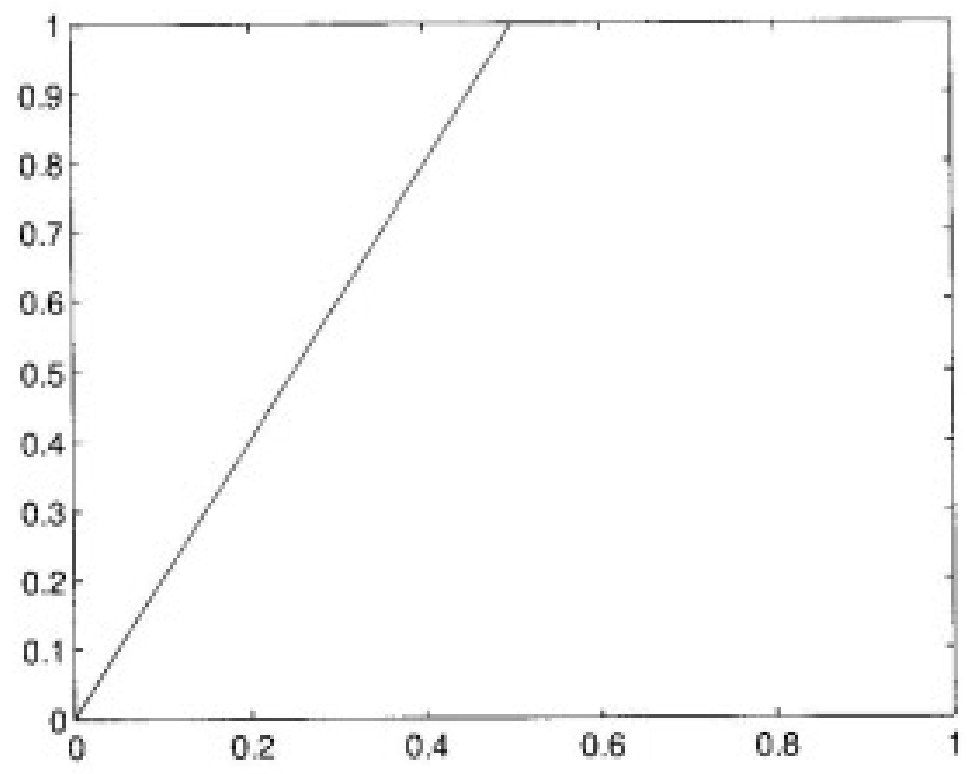

Fig. 1 Lorenz curve for the simple binary model $D=\{r=(1,1,1,1,0,0), s=(0,0,1,1,0,1)\}$, encoded as $\left[\begin{array}{llllll}1 & 1 & 1 & 0 & 0 & 0\end{array}\right]$ 


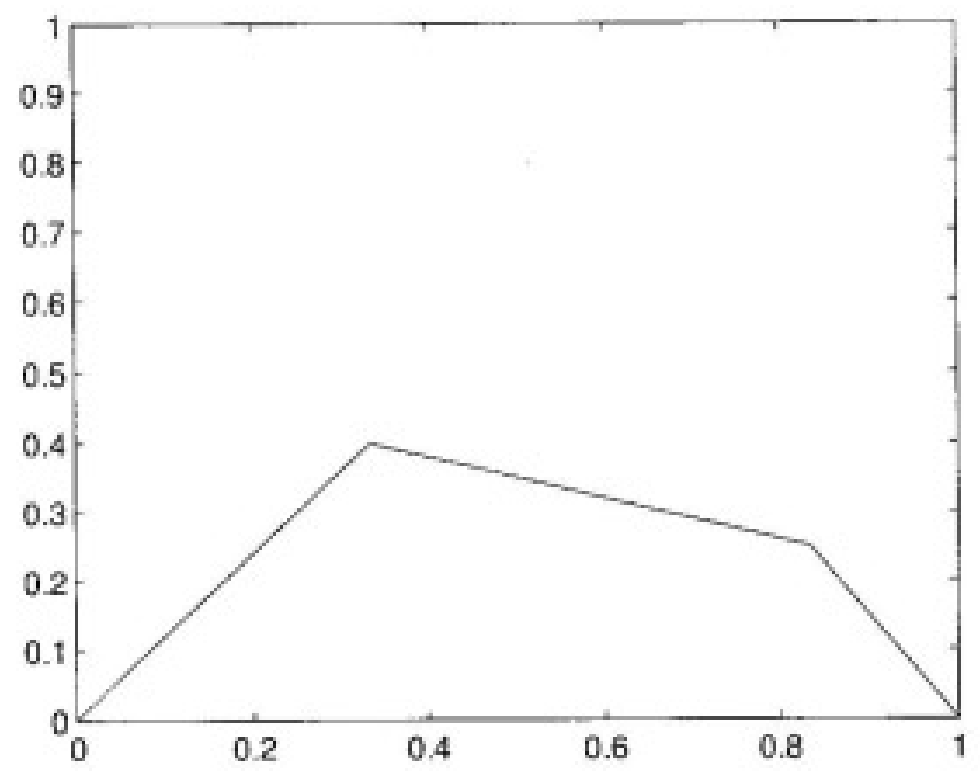

Fig.2 $D=\{r=(1,1,1,1,0,0), s=(0,0,1,1,0,1)\}$, is first rewritten as:

$D^{*}=\left\{r^{*}=(1,1,1,1,1,0), s^{*}=(0,0,1,1,1,1)\right\}$, and then represented by the similarity Lorenz curve joining the points $(0,0)-(2 / 6,8 / 20)-(5 / 6,5 / 20)-(1,0)$. 


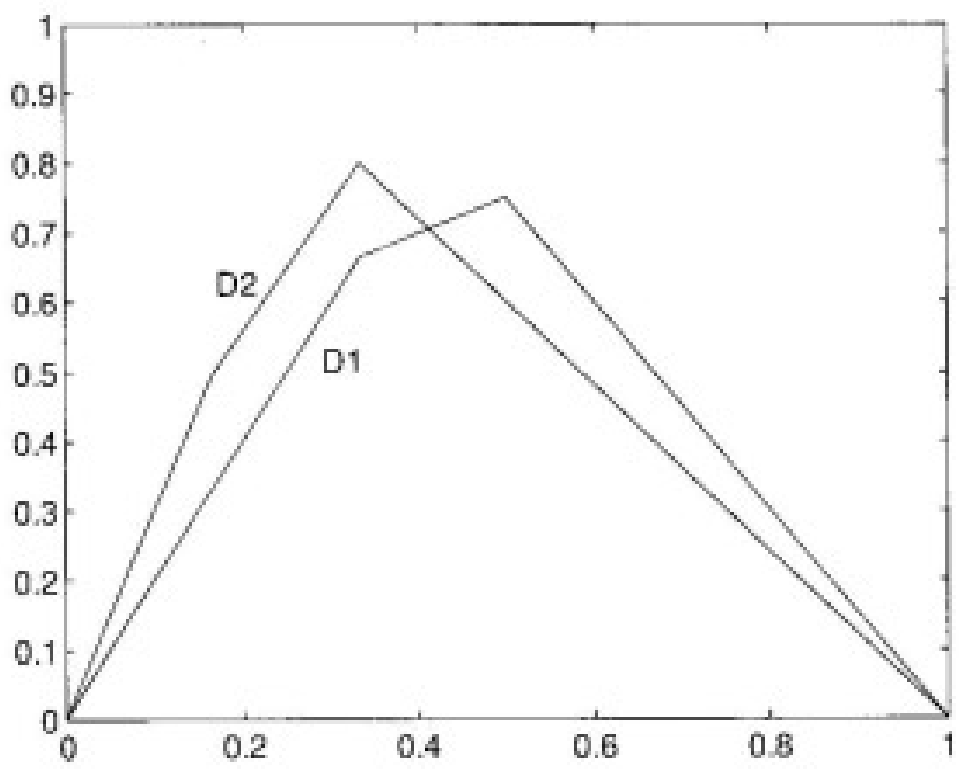

Fig.3 D1 $=\left\{r_{1}=(1,1,1,0,0,0), s_{1}=(1,0,0,1,1,1)\right\}$ and $D 2=\left\{r_{2}=(1,1,1,1,1,0), s_{2}=(1,0,0,0,0,1)\right\}$ are incomparable in the reduction model, because their Lorenz curves cross 


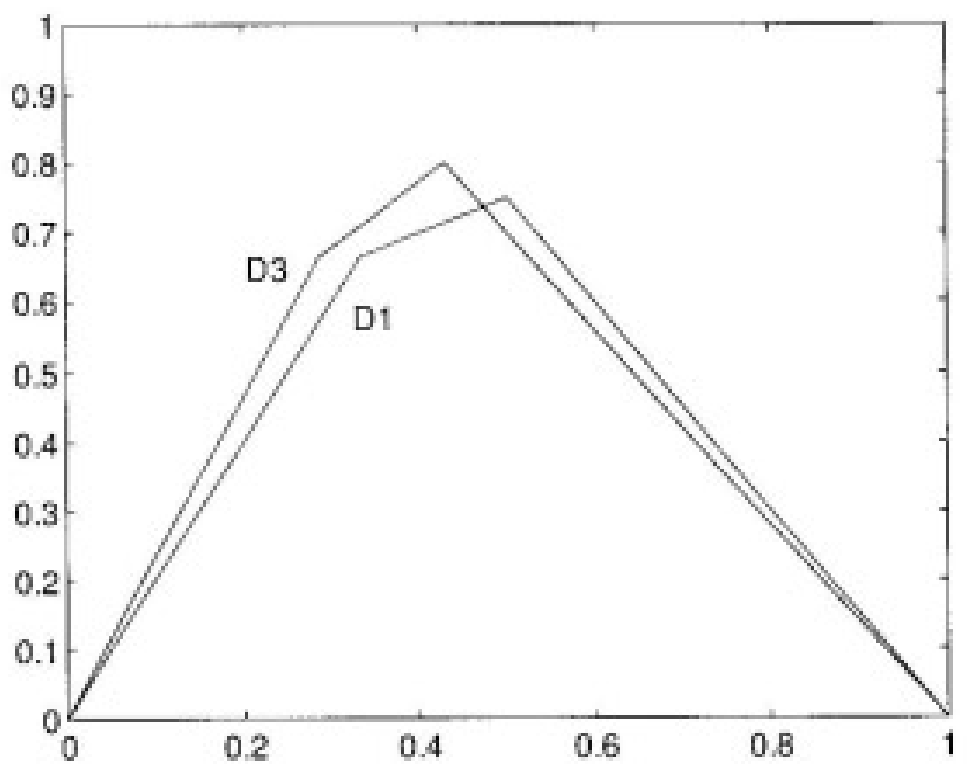

Fig. 4 The transformation of $D 1=\left\{r_{1}=(1,1,1,0,0,0), s_{1}=(1,0,0,1,1,1)\right\}$ to $D 3-\left\{r_{3}-(1,1,1,0,0,0,0), s_{2}=(1,0,0,1,1,1,1)\right\}$ (i.e. adding a $\left.(0-1)\right) ;$ their Lorenz curves cross, showing that D1 and D3 are not intrinsically comparable. 


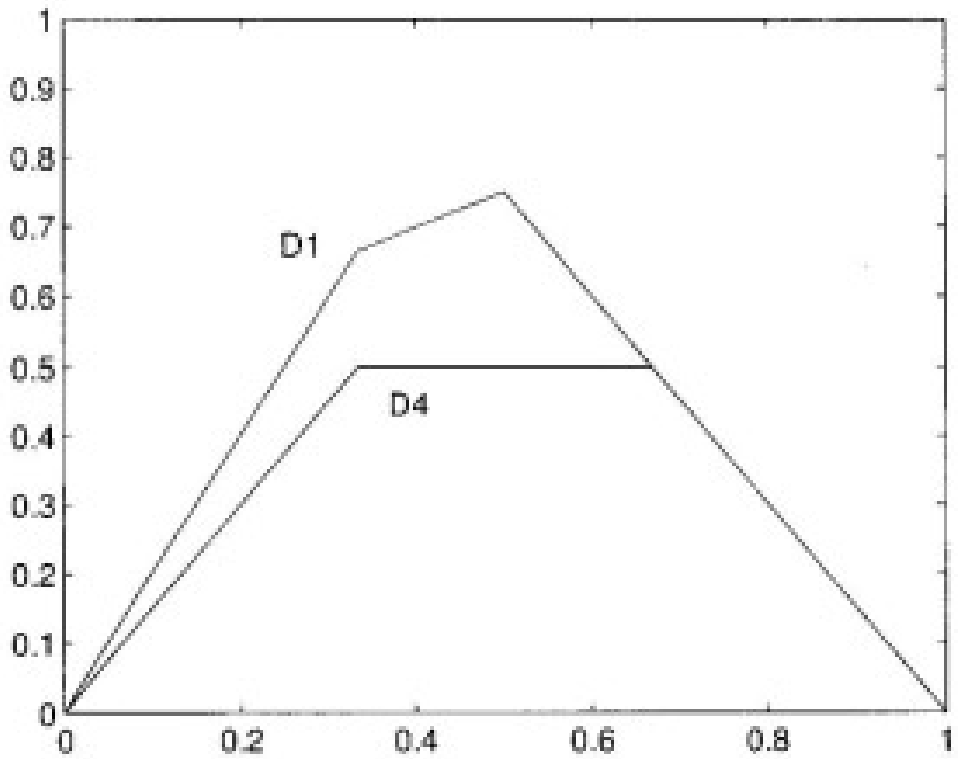

Fig.5 Transformation of $D 1=\left\{r_{1}=(1,1,1,0,0,0), s_{1}=(1,0,0,1,1,1)\right\}$ to $D 4=\left\{r_{4}=(1,1,1,0,0,1), s_{4}=(1,0,0,1,1,1)\right\}$, illustrating that the reduction approach satisfies requirement $\mathrm{P} 4$ 